\title{
Disordered C-terminal Domain of Tyrosyl Transfer-RNA Synthetase: Evidence for a Folded State
}

\section{Valérie Guez-Ivanier and Hugues Bedouelle*}

Groupe d'Ingénierie des

Protéines (CNRS URA1129)

Unité de Biochimie Cellulaire

Institut Pasteur, 28 rue du

Docteur Roux, 75724, Paris

Cedex 15, France

\begin{abstract}
The C-terminal domain (residues 320 to 419) of tyrosyl-tRNA synthetase from Bacillus stearothermophilus (Bst-TyrRS) is necessary for the binding of tRNA $^{\text {Tyr }}$ but disordered in the crystal structure. Four different criteria showed that the isolated C-terminal domain of Bst-TyrRS was at least partially folded in solution. Its spectrum of circular dichroism was compatible with a high content of secondary structure elements $(56 \%$ of its residues) and these structural elements disappeared in $7.5 \mathrm{M}$ urea. It was unfolded by urea along a unique transition, around $6.0 \mathrm{M}$, according to the variations in the fluorescence of its tyrosine residues and in its electrophoretic mobility through transverse gradient gels. It was denatured by heat with a temperature of half-precipitation in 30 minutes that was equal to $67.9^{\circ} \mathrm{C}$ and close to the Bst-TyrRS one, $68.7^{\circ} \mathrm{C}$. Its transitions of denaturation by urea or temperature were weakly cooperative. The C-terminal domains of the TyrRSs from Escherichia coli (Eco-TyrRS) and B. stearothermophilus could be genetically exchanged without a significant loss of aminoacylation activity. A hybrid between the N-terminal domain of Bst-TyrRS and the C-terminal domain of Eco-TyrRS was precipitated by heat in 30 minutes following two transitions: $83 \%$ of the molecules were precipitated with a temperature of half-transition $\left(51.6^{\circ} \mathrm{C}\right)$ close to the Eco-TyrRS one $\left(48.6^{\circ} \mathrm{C}\right)$. The remainder was precipitated with a temperature of half-transition $\left(65.5^{\circ} \mathrm{C}\right)$ close to the Bst-TyrRS one $\left(67.2^{\circ} \mathrm{C}\right)$ or that of its $\mathrm{N}$-terminal domain $\left(68.0^{\circ} \mathrm{C}\right)$. These results showed that the C-terminal domain of Eco-TyrRS could undergo a transition from a soluble active conformation to an insoluble one. The denaturations of Bst-TyrRS and of its N-terminal domain by urea occurred with two successive transitions, around $4 \mathrm{M}$ and $6 \mathrm{M}$, and thus according to a complex mechanism.

(C) 1996 Academic Press Limited
\end{abstract}

Keywords: aminoacyl transfer-RNA synthetase; hybrid protein; nucleic-acid binding protein; protein folding; structural disorder that recognize a specific sequence or structure, e.g. transcription factors (Frankel \& Kim, 1991; Frankel, 1992).

Tyrosyl-tRNA synthetase from Bacillus stearothermophilus (Bst-TyrRS) is a dimeric protein. Each monomer is composed of three domains in the crystal structure: an $\alpha / \beta$ domain (residues 1 to 220 ), an $\alpha$-helical domain (248 to 319) and a C-terminal domain (320 to 419), for which it was not possible to trace the polypeptide chain. Difference maps show a continuous volume of electron density $\left(1500 \AA^{3}\right.$ ), well above the noise level, which might correspond to part of the C-terminal domain. This region is adjacent to the $\alpha$-helical domain and the tyrosyl-adenylate (Tyr-AMP) binding site. Within the crystals, the ordered N-terminal domains 
(residues 1 to 319) pack tightly together to form layers with a maximum thickness of $60 \AA$ that are separated by $20 \AA$. The regions of disordered density occupy the gaps between these layers (Brick et al., 1989). The architecture of the $\alpha / \beta$ domain is typical of the class I aminoacyl-tRNA synthetases (Schimmel et al., 1993).

Bst-TyrRS catalyses the aminoacylation of tRNA $^{\text {Tyr }}$ in a two-step reaction. Tyrosine is first activated with ATP to form Tyr-AMP, then this intermediate is attacked by tRNA ${ }^{\text {Tyr }}$ to form tyrosyl-tRNA $^{\text {Tyr }}$ (Fersht, 1987; Avis et al., 1993). The N-terminal domain of Bst-TyrRS contains the dimerization interface and is sufficient to catalyse the formation of Tyr-AMP. The C-terminal domain is necessary for the binding of tRNA ${ }^{\mathrm{Tyr}}$ (Waye et al., 1983). Six of the 12 basic residues in this domain, at positions 368, 371, 407, 408, 410 and 411, are strongly involved in the interaction between Bst-TyrRS and tRNA ${ }^{\mathrm{Tyr}}$ (Bedouelle \& Winter, 1986; Bedouelle et al., 1993). TyrRS from Escherichia coli (Eco-TyrRS) is 58\% identical in amino acid sequence with Bst-TyrRS (Winter et al., 1983). The former is a thermolabile protein whereas the latter is thermostable (Guez-Ivanier et al., 1993).

To test whether the C-terminal domains of Bst-TyrRS and Eco-TyrRS are folded or unfolded in solution, we compared the behaviours of mutant and hybrid enzymes in experiments of thermal precipitation. We purified the isolated C-terminal domain of Bst-TyrRS to homogeneity. We analysed its unfolding by urea through gel electrophoresis and spectrofluorimetry, and its content in secondary structures by circular dichroïsm (CD) spectroscopy.

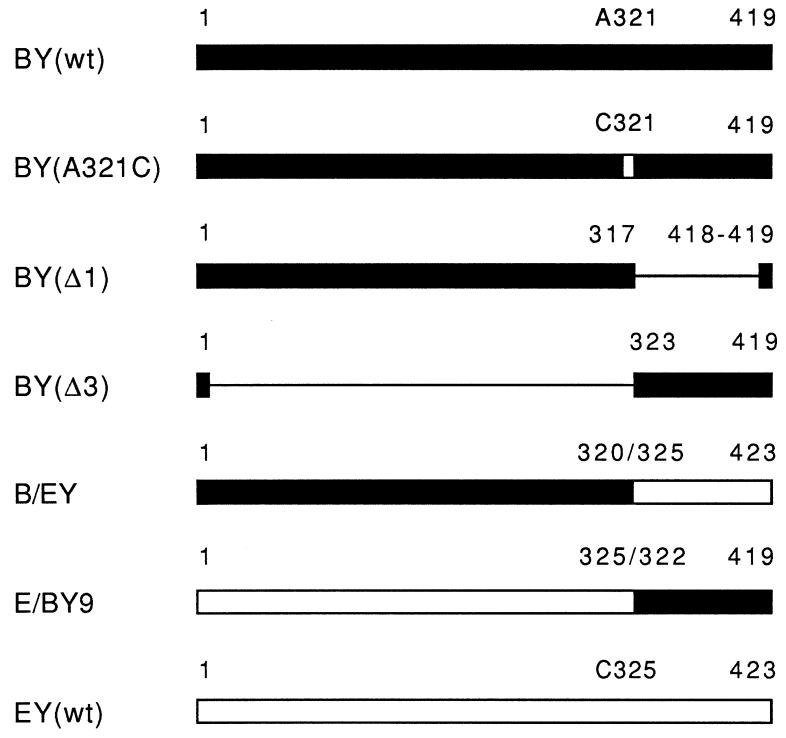

Figure 1. TyrRS derivatives compared in this study. B, B. stearothermophilus; E, E. coli; Y, tyrosyl-tRNA synthetase; wt, wild-type; A321C, change of residue Ala321 into Cys. The filled and open boxes represent sequence segments from B. stearothermophilus and E. coli, respectively. The lines between boxes represent segments that were deleted. The limits of the segments in the two sequences are indicated. See Table 1 for references.

\section{Results}

\section{Production and purification of the TyrRS derivatives}

The mutant and hybrid TyrRSs that we studied are depicted in Figure 1. The genes that coded for

Table 1. Phage and plasmid strains

\begin{tabular}{|c|c|c|}
\hline Strain & Relevant characteristics & References/Source \\
\hline \multicolumn{3}{|l|}{ A. M13 phages } \\
\hline M13mp19(4am) & Like M13mp19 but with an amber mutation in gene 4 & Carter et al. (1985) \\
\hline M13-BY(M24) & B. stearothermophilus tyrSp $p^{+}$tyrS $S^{+}$; codes for Bst-TyrRS & Waye \& Winter (1986) \\
\hline M13-BY(M24.89) & $\begin{array}{l}\text { Like M13-BY(M24) but tyrSp is deleted of residues }-44 \text { to }-98 \text {; } \\
\text { overproduces Bst-TyrRS }\end{array}$ & Waye \& Winter (1986) \\
\hline M13(4am)-BY(M24.89) & $\begin{array}{l}\text { Like M13-BY(M24.89) but with an amber mutation in gene } \\
4 \text { of M13 }\end{array}$ & Carter et al. (1985) \\
\hline $\operatorname{M13-BY}(\Delta 1)$ & $\begin{array}{l}\text { Like M13-BY(M24.89) but codes for a TyrRS with a deletion, } \\
\Delta 1 \text {, of amino acid residues } 318 \text { to } 417\end{array}$ & Waye et al. (1983) \\
\hline $\operatorname{M13-BY}(\Delta 3)$ & $\begin{array}{l}\text { Like M13-BY(M24) but codes for a TyrRS with a deletion, } \\
\Delta 3 \text {, of amino acid residues } 2 \text { to } 322\end{array}$ & G. Winter \\
\hline M13-BY(BsmI) & $\begin{array}{l}\text { Like M13-BY(M24.89) but with a BsmI site at codons } 320 \text { to } 322 \\
\text { of tyrS; codes for a mutant TyrRS (Ala321 } \rightarrow \text { Cys) }\end{array}$ & Guez-Ivanier et al. (1993) \\
\hline M13-B/EY & $\begin{array}{l}\text { Like M13-BY(M24.89) but codes for a hybrid TyrRS comprising } \\
\text { residues } 1 \text { to } 320 \text { of Bst-TyrRS and residues } 325 \text { to } 423 \text { of Eco-TyrRS }\end{array}$ & This work \\
\hline \multicolumn{3}{|l|}{ B. Plasmids } \\
\hline pEMBL8 $^{+}$, pEMBL9 $^{+}$ & $b l a^{+}$, carry the intergenic region of phage $\mathrm{f} 1$ & Dente \& Cortese (1987) \\
\hline pBR322-EY & E. coli tyrSp ${ }^{+}$tyrS ${ }^{+}$; bla ; codes for Eco-TyrRS & Barker (1982) \\
\hline pEMBL8-EY & E. coli tyrSp ${ }^{+}$tyrS $S^{+}$; la $^{+}$; codes for Eco-TyrRS & Bedouelle et al. (1990) \\
\hline pEMBL9-BY(Ptac) & Ptac Bst-tyrS'; bla ; codes for Bst-TyrRS & Bedouelle et al. (1990) \\
\hline pEMBL-E/BY9 & $\begin{array}{l}\text { Like pEMBL8-EY but codes for a hybrid TyrRS comprising } \\
\text { residues } 1 \text { to } 325 \text { of Eco-TyrRS and residues } 322 \text { to } 419 \text { of Bst-TyrRS }\end{array}$ & Guez-Ivanier et al. (1993) \\
\hline \multicolumn{3}{|c|}{$\begin{array}{l}\text { Bst-tyrS and Eco-tyrS, genes of the tyrosyl-tRNA synthetases from B. stearothermophilus and E. coli; tyrSp, promoter } \\
\text { of tyrS; bla, B-lactamase gene. The tyrS gene had the same orientation as lacZ in the phages and as bla in the } \\
\text { plasmids. }\end{array}$} \\
\hline
\end{tabular}


$\begin{array}{lllllllllll}1 & 2 & 3 & 4 & 5 & 6 & 7 & 8 & 9 & 10 & 11\end{array}$
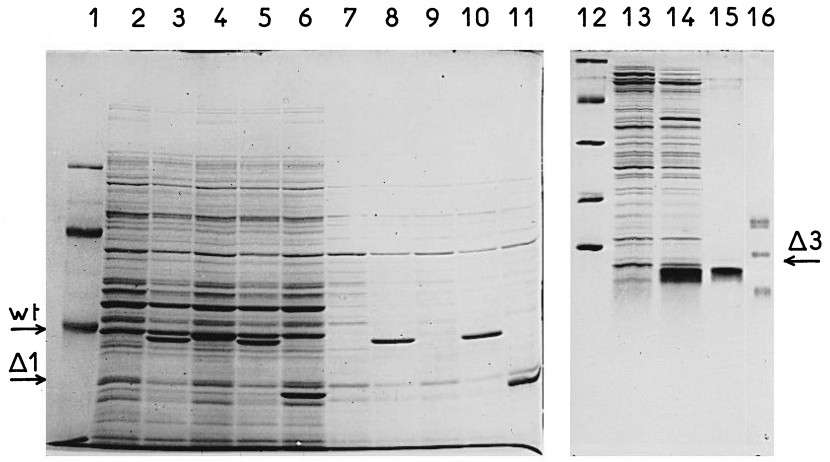

Figure 2. Expression of TyrRS derivatives from M13 phages. Lanes 1 to 11 and 12 to 16, SDS-containing gels at $8 \%$ and $17 \%$ polyacrylamide, respectively. Lanes 2 to 6 , soluble extracts were loaded onto the gels without heat treatment; lanes 7 to 11, 13 and 14, the proteins of the extracts were thermoprecipitated during 30 minutes at $56^{\circ} \mathrm{C}$ before electrophoresis (Materials and Methods). The extracts were prepared from TG2 bacteria infected with the following phages: lanes 2, 7 and 13, M13mp19(4am); lanes 3 and 8, M13(4am)-BY(M24.89); lanes 4 and 9, M13-B/EY; lanes 5 and 10, M13-BY(BsmI); lanes 6 and 11, $\operatorname{M13-BY}(\Delta 1)$; lane $14, \operatorname{M13-BY}(\Delta 3)$. The samples corresponded to $30 \mu \mathrm{l}$ of bacterial culture at $A_{600 \mathrm{~nm}}=1.0$ for lanes 2 to 11 and to $180 \mu \mathrm{l}$ for lanes 13 and 14 . Lane 15, purified Bst-TyrRS $(\Delta 3)(5 \mu \mathrm{g})$. Molecular mass markers (in $\mathrm{kDa}): 31,45,66$ and 97 in lane 1; 14.5, 21.5, 31, 45 and 66 in lane 12; 8.1, 14.4 and 16.9 in lane 16. The expected molecular masses for the TyrRS derivatives, calculated from their amino acid sequences, were the following (in Da): 47,302 for Bst-TyrRS(wt), 47,334 for BstTyrRS(A321C), 47,555 for Bst/Eco-TyrRS, 36,310 for $\operatorname{Bst-TyrRS}(\Delta 1)$ and 10,862 for Bst-TyrRS( $\Delta 3)$. The positions of Bst-TyrRS(wt), Bst-TyrRS( $\Delta 1)$ and Bst-TyrRS( $\Delta 3)$ are indicated. The proteins were stained with Coomassie blue.

derivatives of Bst-TyrRS were carried by derivatives of phage M13-BY(M24). Those that coded for derivatives of Eco-TyrRS were carried by derivatives of phagemid pEMBL8-EY (Table 1).

We analysed the production of the Bst-TyrRS derivatives by two methods: electrophoresis of the proteins through SDS/polyacrylamide gels and active site titration. These analyses were performed on soluble extracts of strain TG2, used as a cellular host. The cells that were infected by the derivatives of M13-BY(M24) overproduced polypeptides that had apparent molecular masses close to those expected for the monomers of the Bst-TyrRS derivatives, in gels stained with Coomassie blue (Figure 2). These polypeptides were the major species of the soluble extracts that reacted with an anti-TyrRS serum in Western blots (not shown, but see Figure 4(a)). They were not produced when the cells were infected by the parental vector M13mp19(4am), which does not carry the tyrS gene (Figures 2 and 4(a)). Bst/Eco-TyrRS was precipitated by a heat treatment of 30 minutes at $56^{\circ} \mathrm{C}$, whereas Bst-TyrRS(wt), Bst-TyrRS(A321C), $\operatorname{Bst-TyrRS}(\Delta 1)$ and $\operatorname{Bst-TyrRS}(\Delta 3)$ were resistant to this treatment (Figure 2). Active site titration showed that Bst/Eco-TyrRS, Bst-TyrRS(wt),
Bst-TyrRS(A321C) and Bst-TyrRS( $\Delta 1)$ were produced in similar amounts (respectively $128 \%, 100 \%$, $80 \%$ and $50 \%$ the amount of TyrRS (wt)) and that these proteins were at least partially active for the formation of Tyr-AMP.

We used two observations to purify Bst$\operatorname{TyrRS}(\Delta 3)$. This protein was resistant to heat treatment at $56^{\circ} \mathrm{C}$, contrary to most E. coli proteins. It was not retained by an anion-exchange resin at $\mathrm{pH} 7.5$, which is close to the value of its isoelectric point, estimated from its amino acid sequence. We purified Bst-TyrRS(wt) and Bst-TyrRS( $\Delta 1)$, which are also thermostable, as described in Materials and Methods. Some of our experiments on Bst-TyrR$S(w t)$, Eco-TyrRS(wt) and their derivatives were performed on soluble extracts of the producing cells, others on purified proteins.

\section{Activity of the TyrRSs}

We measured the activities of the different TyrRSs, in soluble extracts of the producing cells, for the aminoacylation of tRNA ${ }^{\text {Tyr }}$ with tyrosine. For each extract, we measured both the concentration of TyrRS active sites and the rate of tyrosylation of crude $E$. coli tRNA so that the rates are expressed in $\mathrm{s}^{-1}$ (Table 2). Bst-TyrRS(wt) and Bst/Eco-TyrRS charged tRNA ${ }^{\mathrm{Tyr}}$ with similar rates, which showed that both the N-terminal domain from Bst-TyrRS and the C-terminal domain from Eco-TyrRS had an active conformation in their hybrid.

\section{Thermal precipitation of active TyrRSs}

We compared the behaviours of the TyrRS derivatives in experiments of irreversible thermal precipitation. We expected that hybrid Bst/EcoTyrRS would be precipitated at the same temperature as Eco-TyrRS(wt) if its C-terminal domain was folded, and at the same temperature as Bst-TyrR$\mathrm{S}(\mathrm{wt})$ if its C-terminal domain was permanently unfolded. Portions of a soluble extract, prepared from producing cells, were heated for 30 minutes at varying temperatures. After elimination of the protein precipitate by centrifugation, the concentration of TyrRS in the supernatant was determined by active site titration. The profiles of precipitation as a function of temperature had a sigmoid shape and showed a unique and steep transition from a soluble to an insoluble state for all the TyrRS derivatives tested, except the profile for Bst/EcoTyrRS, which showed two transitions (Figure 3). Table 2A gives the temperatures of half-transitions that we deduced from these profiles and called $t_{1 / 2}$. Note that $t_{1 / 2}$ is not a thermodynamic parameter because precipitation is an irreversible process.

\section{Thermal precipitation of the C-terminal domain}

$\operatorname{Bst-TyrRS}(\Delta 3)$ only comprised the C-terminal domain of Bst-TyrRS and had no measurable catalytic activity. We therefore used its antigenic activity to measure its concentration in cellular 
extracts. We compared the behaviours of Bst$\operatorname{TyrRS}(\Delta 3)$ and Bst-TyrRS(wt) in experiments of thermal precipitation that were performed as described in the previous section, except that the concentrations of soluble TyrRS were measured by revelation of Western blots with an anti-TyrRS serum and quantification of the protein bands (Figure 4(a); Materials and Methods). The profiles of precipitation as a function of temperature had sigmoid shapes (Figure 4(b)). The temperatures of half precipitation $\left(t_{1 / 2}\right)$ were close for $\operatorname{Bst-TyrRS}(\Delta 3)$ and Bst-TyrRS(wt), but the transition between the soluble and insoluble states was less cooperative for Bst-TyrRS( $\Delta 3$ ) than for Bst-TyrRS(wt) (Table 2B).

\section{Thermal precipitation of the E. coli proteins}

We performed the experiments of thermal precipitation described above on soluble extracts of bacteria that produced the TyrRS derivatives. It was therefore important to test the effect of

Table 2. Activity and stability of the TyrRS derivatives

\begin{tabular}{lccc}
\hline Protein & $V\left(\mathrm{~s}^{-1}\right)$ & $t_{1 / 2}\left({ }^{\circ} \mathrm{C}\right)$ & $c$ \\
\hline A. Active site titration & & & \\
Bst-TyrRS(wt) & $1.67 \pm 0.09$ & $67.23 \pm 0.03$ & $1.12 \pm 0.04$ \\
Bst-TyrRS(A321C) & $2.43 \pm 0.05$ & $66.90 \pm 0.01$ & $1.96 \pm 0.04$ \\
Bst-TyrRS( $\Delta 1)$ & na & $67.97 \pm 0.02$ & $1.41 \pm 0.03$ \\
Bst/Eco-TyrRS & $1.88 \pm 0.07$ & $51.58 \pm 0.06$ & $0.54 \pm 0.01$ \\
& & $65.45 \pm 0.16$ & $1.26 \pm 0.22$ \\
Eco/Bst-TyrRS9a & $0.70 \pm 0.03$ & $45.22 \pm 0.18$ & $0.51 \pm 0.05$ \\
Eco-TyrRS(wt) & $4.61 \pm 0.11$ & $48.58 \pm 0.01$ & $0.86 \pm 0.05$ \\
B. Gel scanning & & & \\
Bst-TyrRS(wt) & $1.67 \pm 0.09$ & $68.71 \pm 0.11$ & $0.81 \pm 0.06$ \\
Bst-TyrRS $(\Delta 3)$ & na & $67.92 \pm 0.30$ & $0.27 \pm 0.03$ \\
\hline
\end{tabular}

Column 1, The TyrRS derivatives were overproduced from TG2 bacteria, either infected by one of the phages or harbouring one of the phagemids listed in Table 1. M13mp19(4am) and pEMBL9 $^{+}$were used as negative controls of expression. The same values were obtained for Bst-TyrRS(wt) from M13(4am)BY(M24.89) (this work) and from pEMBL9-BY(Ptac) (GuezIvanier et al., 1993). Column 2, Rate of tyrosylation (V) of crude E. coli $\mathrm{tRNA}(5 \mathrm{mg} / \mathrm{ml}, 356 \mathrm{pmol}$ of tyrosine incorporation $/ \mathrm{mg}$ ) by soluble extracts of the overproducing bacteria. The concentration of TyrRS in the extracts was determined by active site titration. If $v$ was the tyrosylation rate by the extract of the overproducing bacteria, $e$ the concentration of TyrRS active sites in the aminoacylation reaction, $v_{0}$ and $e_{0}$ the same parameters for a similar extract prepared from the negative control strain, then $V$ was calculated using the relation $\mathrm{V}=\left(v-v_{0}\right) /\left(e-e_{0}\right)$ to correct the rate for the contribution of the tyrS gene on the bacterial chromosome to the production of TyrRS activity. The average $V$ value and the standard error of at least three different measurements are shown; $e$ was between 0.3 and $1.6 \mathrm{nM}$; the average value of $e / e_{0}$ was equal to 25 ; na, not applicable. Columns 3 and 4 , Temperatures of half-advancement $\left(t_{1 / 2}\right)$ and cooperativity indices $(c)$ of the transitions in the experiments of thermal precipitation, with their standard errors in the curve fits. After precipitation, the concentration of soluble TyrRS was measured by active site titration (part A of the Table, and see Figure 3 ) or by gel scanning (part B of the Table, and see Figure 4). The following equation was fitted to the experimental data: $Y=100\left(m /\left(1+\exp \left(c_{1}\left(t-t_{1}\right)\right)\right)+(1-m) /\left(1+\exp \left(c_{2}\left(t-t_{2}\right)\right)\right)\right)$, where $Y$ is the relative concentration of soluble TyrRS, in percentage, and $t$ is temperature. We found $m=0.83 \pm 0.01$ for the thermal precipitation of Bst/Eco-TyrRS. We took $m=1$ in all the other cases.

${ }^{a}$ Data from Guez-Ivanier et al. (1993).

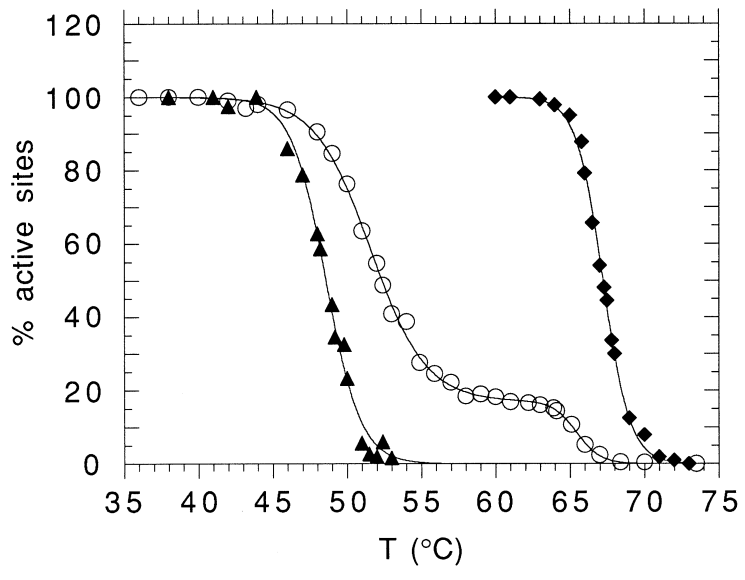

Figure 3. Thermal precipitation of Bst/Eco-TyrRS. The TyrRS derivatives were produced by TG2 bacteria, infected with M13(4am)-BY(M24.89), M13-BY( $\Delta 1)$, M13$\mathrm{B} / \mathrm{EY}$, or harbouring phagemid pEMBL8-EY. Portions of a cellular extract were heated at varying temperatures during 30 minutes, the protein precipitates were eliminated and the concentrations of soluble TyrRS were determined by active site titration (Materials and Methods): $100 \%$ active sites corresponds to the concentration of TyrRS in the extract before heating. ( $)$ Bst-TyrRS(wt); (A) Eco-TyrRS(wt); (○) Bst/Eco-TyrRS. The profile for Bst-TyrRS $(\Delta 1)$ was nearly identical with that for Bst-TyrRS(wt); it was not represented for clarity. The data for Eco-TyrRS(wt) are from Guez-Ivanier et al. (1993). The curves were obtained by fitting the equations described in the legend to Table 2 to the data.

a thermal treatment on the bulk of the E. coli proteins, as a control. We used a soluble extract of TG2 bacteria, infected with the parental vector M13mp19(4am). After the thermal precipitation, the total concentration of $E$. coli soluble proteins was measured by the method of Bradford (1976). The precipitation of the E. coli proteins in 30 minutes was a simple exponential function of temperature, with $t_{1 / 2}=46^{\circ} \mathrm{C}$ (Figure 5). The experimental values obtained for temperatures varying between $40^{\circ} \mathrm{C}$ and $62^{\circ} \mathrm{C}$ could be extrapolated to $100 \%$ and $10 \%$ proteins remaining soluble after 30 minutes at $37.3^{\circ} \mathrm{C}$ and $65.4^{\circ} \mathrm{C}$, respectively. Thus, the profile of thermal precipitation for the bulk of the E. coli proteins was clearly different from those obtained for the TyrRS derivatives.

\section{Denaturation of Bst-TyrRS and its domains with urea: analysis by gel electrophoresis}

We compared the effect of urea on the conformations of Bst-TyrRS(wt), Bst-TyrRS( $\Delta 1)$ and Bst-TyrRS $(\Delta 3)$ by electrophoresis of the purified proteins through polyacrylamide gels, perpendicularly to a urea gradient $(0$ to $8 \mathrm{M})$. The three proteins underwent a transition between a form that was present at low concentration of urea and migrated faster, and a form that was present at high concentration of urea and migrated slower. The transition was discontinuous for Bst-TyrRS(wt), around $6.4 \mathrm{M}$ urea, and $\operatorname{Bst}-\operatorname{TyrRS}(\Delta 1)$, around 


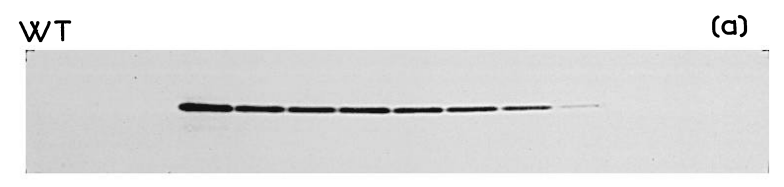

$\Delta 3$
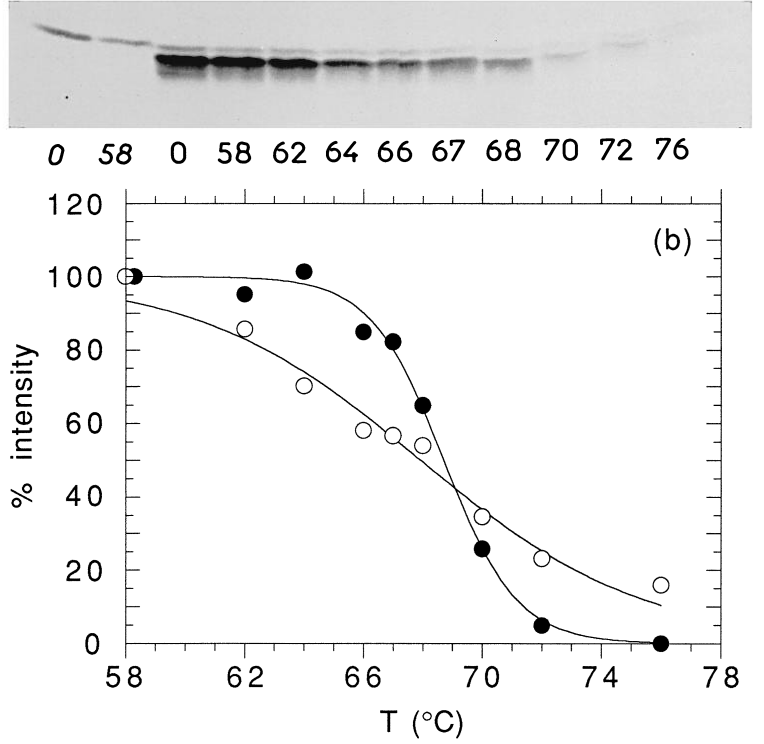

Figure 4. Thermal precipitation of $\operatorname{Bst-TyrRS}(\Delta 3)$. (a) TG2 bacteria were infected with M13(4am)$\mathrm{BY}(\mathrm{M} 24.89)$ (top row), M13-BY( $\Delta 3)$ (bottom row) and M13mp19 as a control (first two lanes of each row). Portions of a cellular extract were heated at the indicated temperatures during 30 minutes, the protein precipitates were eliminated by centrifugation and the supernatants were analysed by SDS/polyacrylamide gel electrophoresis $(8 \%$ and $17 \%$ polyacrylamide for the top and bottom rows, respectively). The TyrRS derivatives were revealed by Western blotting and immuno-staining with an anti-TyrRS serum. (b) The intensities of the protein bands on the membranes were quantified with an image analyser: $100 \%$ intensity corresponds to the sample heated at $58^{\circ} \mathrm{C}$. (O) Bst-TyrRS(wt); (O) Bst-TyrRS $(\Delta 3)$.

5.6 M, as previously reported (Carter et al., 1986). It was continuous and occurred above $5.8 \mathrm{M}$ urea (Figure 6) for Bst-TyrRS( $\Delta 3)$. We obtained similar profiles of electrophoresis from samples containing native proteins and samples containing proteins that had been denatured with high concentrations of urea beforehand. Thus the denaturation with urea was reversible in the conditions of the electrophoresis. We estimated the free energy of unfolding in the absence of urea for $\operatorname{Bst-TyrRS}(\Delta 3)$ from its profile of electrophoresis, by using a published method (Hollecker \& Creighton, 1982). We found a value of this free energy, $\Delta G\left(\mathrm{H}_{2} \mathrm{O}\right)$, equal to $7.0 \mathrm{kcal} / \mathrm{mol}$.

\section{Denaturation of Bst-TyrRS and its domains with urea: analysis by fluorimetry}

We followed the denaturation of Bst-TyrRS(wt) and its truncated derivatives by urea at $25^{\circ} \mathrm{C}$, using spectrofluorimetry. Bst-TyrRS(wt) and BstTyrRS $(\Delta 1)$ each contain six tryptophan residues,

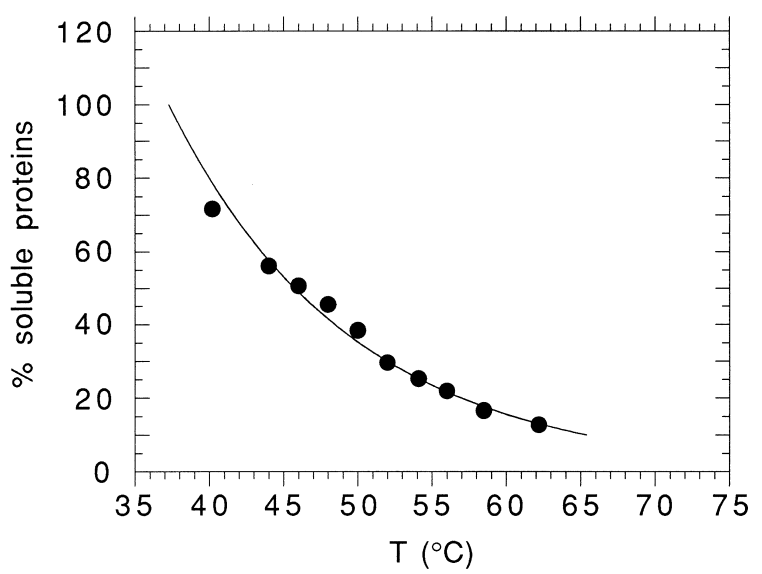

Figure 5. Thermal precipitation of the E. coli proteins. TG2 bacteria were infected with the parental phage M13mp19. Portions of a cellular extract were heated at varying temperatures during 30 minutes, the protein precipitates were eliminated by centrifugation and the concentrations of soluble proteins were determined by the Bradford method. The percentage of soluble proteins is plotted as a function of the temperature of heating; $100 \%$ corresponds to the unheated sample.

whereas Bst-TyrRS $(\Delta 3)$ contains none. The three proteins contain 14, 11 and 4 tyrosine residues, respectively. The fluorescence of both tryptophan and tyrosine are excited at a wavelength of $278 \mathrm{~nm}$, whereas only tryptophan is excited at $295 \mathrm{~nm}$. Accordingly, we found that the purified Bst$\operatorname{TyrRS}(\Delta 3)$ did not emit fluorescence between 310 and $400 \mathrm{~nm}$ when excited at $295 \mathrm{~nm}$, in contrast to the two other proteins. The emission spectra of the proteins upon excitation at $278 \mathrm{~nm}$ showed that the highest ratios of fluorescence intensity between native (0 M urea) and denaturing $(8 \mathrm{M})$ conditions were obtained for the following wavelength of emission: $330 \mathrm{~nm}$ for Bst-TyrRS(wt) and Bst$\operatorname{TyrRS}(\Delta 1)$, and $303 \mathrm{~nm}$ for Bst-TyrRS $(\Delta 3)$.

We determined the time needed by the different conformations of Bst-TyrRS $(\Delta 1)$ to reach equilibrium, after dilutions of the protein in $0 \mathrm{M}, 3 \mathrm{M}$ and $5 \mathrm{M}$ urea. The ratio of the fluorescence intensities, measured at $3 \mathrm{M}$ and $0 \mathrm{M}$ urea (or at $5 \mathrm{M}$ and $0 \mathrm{M}$ ), decreased until eight hours of incubation then remained stable between 8 and 18 hours. We thus let the proteins equilibrate during 12 hours in different concentrations of urea before performing the fluorescence measurements.

The denaturation profiles for Bst-TyrRS(wt) and Bst-TyrRS $(\Delta 1)$ showed two transitions, whereas the profile for Bst-TyrRS $(\Delta 3)$ showed only one. Denaturation was reversible, since the same profiles were obtained from native proteins or from proteins that had been denatured in $8 \mathrm{M}$ urea beforehand (Figure 7). Table 3 gives the parameters of the different transitions. The maximum of fluorescence emission for Bst-TyrRS(wt) and Bst-TyrRS( $\Delta 1)$ shifted from $340 \mathrm{~nm}$ at low concentration of urea, to $350 \mathrm{~nm}$ at high concentration. The shift occurred mainly during the second transition (around $6.2 \mathrm{M}$ and 5.6 $\mathrm{M}$ urea, respectively). We did not observe such 


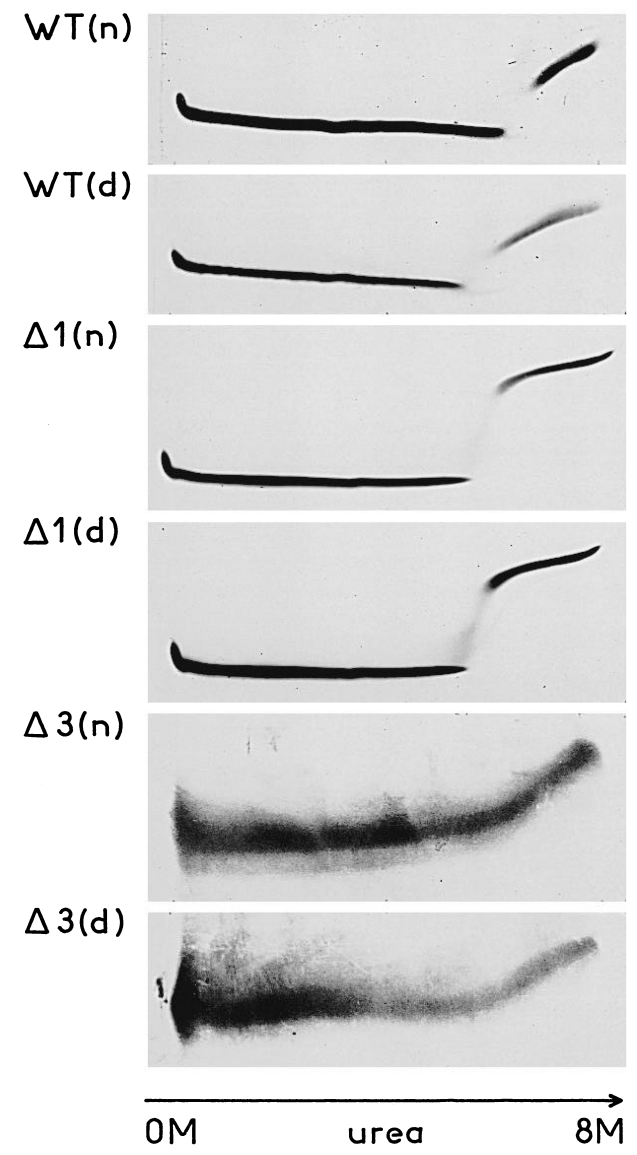

Figure 6. Urea-induced unfolding of Bst-TyrRS(wt) and its $\Delta 1$ and $\Delta 3$ derivatives, as monitored by electrophoresis. The purified proteins were applied across the top of gels containing a transverse gradient of urea (0 to $8 \mathrm{M})$ and a compensatory gradient of polyacrylamide, 10 to $7.5 \%$ for Bst-TyrRS(wt) and Bst-TyrRS( $\Delta 1), 15$ to $11 \%$ for Bst$\operatorname{TyrRS}(\Delta 3)$. They were applied either in a native form (n) or after denaturation during three hours in about $8 \mathrm{M}$ urea (d), and in identical amounts in both conditions: wt, $22 \mu \mathrm{g}$ at $1.5 \mu \mathrm{M} ; \Delta 1,24 \mu \mathrm{g}$ at $1.6 \mu \mathrm{M}$ and $\Delta 3,9 \mu \mathrm{g}$ at $5.6 \mu \mathrm{M}$. After electrophoresis, Bst-TyrRS(wt) and $\operatorname{Bst-TyrRS}(\Delta 1)$ were stained with Coomassie blue; Bst-TyrRS $(\Delta 3)$ was revealed by Western blotting and immuno-staining with an anti-TyrRS serum. Staining of Bst-TyrRS $(\Delta 3)$ with silver gave similar profiles (not shown).

a shift for Bst-TyrRS( $\Delta 3)$, which did not contain tryptophan residues. We estimated the free energy of unfolding in the absence of urea for Bst$\operatorname{TyrRS}(\Delta 3)$ from its denaturation profile by using the relation $\Delta G=\Delta G\left(\mathrm{H}_{2} \mathrm{O}\right)-m$ [urea] (Pace et al., 1989). We found $\Delta G\left(\mathrm{H}_{2} \mathrm{O}\right)=5.1( \pm 0.4) \mathrm{kcal} \mathrm{mol}^{-1}$ and $m=0.85( \pm 0.05) \mathrm{kcal} \mathrm{mol}^{-1} \mathrm{M}^{-1}$.

\section{Circular dichroism spectra of the C-terminal domain}

The far-UV circular dichroism spectra for Bst-TyrRS $(\Delta 3)$, recorded either in the absence or in the presence $(7.5 \mathrm{M})$ of urea, are presented in Figure 8 . The spectrum of the native protein showed a strongly positive molar ellipticity below $200 \mathrm{~nm}$ and a strongly negative ellipticity above $200 \mathrm{~nm}$, with minima between 207 and $217 \mathrm{~nm}$. Deconvolution of this spectrum showed that it corresponded to $16 \%$ of $\alpha$-helix, $21 \%$ of antiparallel $\beta$-sheet, $6 \%$ of parallel $\beta$-sheet and $13 \%$ of turn. The spectrum of Bst-TyRS $(\Delta 3)$ in $7.5 \mathrm{M}$ urea did not show any ellipticity. These spectra showed that Bst-TyrRS $(\Delta 3)$ had secondary structural elements in its native form, and that it was unfolded in $7.5 \mathrm{M}$ urea.

\section{Discussion}

\section{Identification of Bst-TyrRS( $\Delta 3)$}

Bst-TyrRS $(\Delta 3)$ has no characterized enzymatic activity. We therefore used the following criteria for its identification. It was present in the soluble extract of TG2 when this strain was infected with phage M13-BY $(\Delta 3)$ but absent when TG2 was infected with the parental vector M13-mp19(4am). It had an apparent molecular mass close to its theoretical mass. It reacted with a serum directed against Bst-TyrRS(wt) in Western experiments. It did not bind an anion-exchange resin at a $\mathrm{pH}$ equal to its theoretical pI. It did not emit fluorescent light when excited at $295 \mathrm{~nm}$, in accordance with the absence of tryptophan residues in its sequence.

\section{Folded state of an isolated C-terminal domain}

The following results showed that Bst-TyrRS( $\Delta 3)$, i.e. the isolated C-terminal domain of Bst-TyrRS, was at least partially folded in solution. (1) Its far-UV CD-spectrum showed the existence of secondary structures in $0 \mathrm{M}$ urea, which disappeared at high concentration in urea. (2) Its treatment with increasing concentrations of urea led to a transition (around $6.0 \mathrm{M}$ ) between a form whose tyrosine residues fluoresced strongly and a form whose tyrosine residues fluoresced weakly. (3) Its profile of electrophoresis across a urea gradient gel showed a transition (above $5.8 \mathrm{M}$ ) between a more compact state at low concentration in urea and a less compact state at high concentration. (4) Its profile of thermal precipitation from a cellular extract showed a transition around $68^{\circ} \mathrm{C}$ between soluble and insoluble states. This profile was different from that obtained for the bulk of the soluble cellular proteins (Figures 4 and 5). Thus, the precipitation observed for $\operatorname{Bst}-\operatorname{TyrRS}(\Delta 3)$ was due to a conformational change of this protein; it was not due to the unfolding of the E. coli proteins and their aggregation with $\operatorname{Bst}-\operatorname{TyrRS}(\Delta 3)$, permanently present in an unfolded conformation.

Bst-TyrRS $(\Delta 3)$ was unfolded at high concentration in urea, as shown by its absence of ellipticity in far-UV CD, its low compactness and the weak fluorescence of its tyrosine residues. The folding and unfolding of Bst-TyrRS $(\Delta 3)$ were fast when compared to the duration of electrophoresis across the urea gradient gel (half-times $<0.01$ the separation time, i.e. $<2.5$ minutes). Indeed, the electrophoretic profile was continuous and identical 

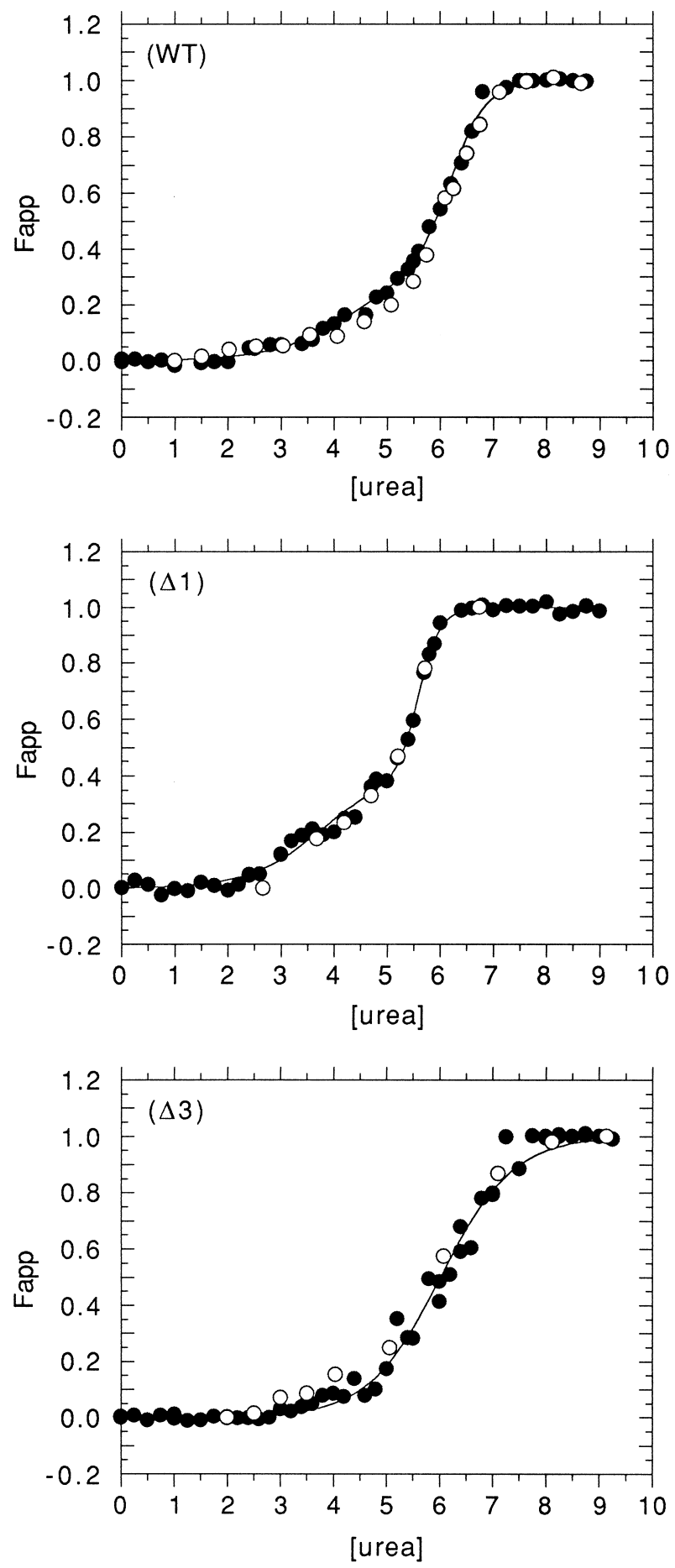

Figure 7. Urea-induced unfolding of Bst-TyrRS(wt) and its $\Delta 1$ and $\Delta 3$ derivatives, as monitored by fluorimetry. The wavelength of the excitation light was $278 \mathrm{~nm}$ and the emission was measured at $330 \mathrm{~nm}$ for Bst-TyrRS(wt) and Bst-TyrRS $(\Delta 1)$, and at $303 \mathrm{~nm}$ for Bst-TyrRS( $\Delta 3) . F_{\text {app }}$, the apparent fraction of denatured protein, is given as a function of the urea concentration (M). $F_{\text {app }}=\left(Y_{\mathrm{n}}-Y_{\text {obs }}\right) /$ $\left(Y_{\mathrm{n}}-Y_{\mathrm{d}}\right)$, where $Y_{\mathrm{n}}$ and $Y_{\mathrm{d}}$ are the intensities of the emitted light for the native and denatured forms respectively. In the transition region, $Y_{\mathrm{n}}$ and $Y_{\mathrm{d}}$ were obtained by linear extrapolations of the values obtained at low and high concentrations of urea. Filled symbols represent values obtained by adding urea to the native profiles were obtained when loading native and unfolded proteins (Creighton, 1980). The transition between the native and denatured states was less cooperative for Bst-TyrRS $(\Delta 3)$ than for Bst-TyrR$S(w t)$ or Bst-TyrRS $(\Delta 1)$ in the experiments of thermal precipitation and of urea denaturation (Tables 2 and 3). The free energy of unfolding for Bst-TyrRS $(\Delta 3)$ at $25^{\circ} \mathrm{C}$ in the absence of urea, determined from fluorimetric measurements, was equal to $\Delta G\left(\mathrm{H}_{2} \mathrm{O}\right)=5.1( \pm 0.4) \mathrm{kcal} \mathrm{mol}^{-1}$. This value was close to that determined from the profiles of electrophoresis across a urea gradient gel at about $15^{\circ} \mathrm{C}, \Delta G\left(\mathrm{H}_{2} \mathrm{O}\right)=7.0 \mathrm{kcal} \mathrm{mol}^{-1}$. The properties of the transitions between the folded and unfolded states of Bst-TyrRS $(\Delta 3)$ (low value of $\Delta G\left(\mathrm{H}_{2} \mathrm{O}\right)$, fast rate of exchange, low cooperativity) suggest that it could be in a molten globule state. This hypothesis would be compatible with the apparent disorder of the C-terminal domain in the crystals of Bst-TyrR$S(w t)$ but remains to be verified experimentally.

\section{Denaturation of Bst-TyrRS and its $\mathrm{N}$-terminal domain}

The profiles of electrophoresis across a urea gradient gel for Bst-TyrRS(wt) and Bst-TyrRS $(\Delta 1)$ showed a unique and discontinuous transition between a fast-migrating state at low concentration in urea and a slow-migrating state at high concentration. The discontinuous character of the transition indicated that the interconversion between the two states was slow in comparison with the time of migration (half-time around 0.1 the migration time, i.e. 25 minutes). The slower migration rates of Bst-TyrRS(wt) and Bst-TyrRS $(\Delta 1)$ during gel electrophoresis and the shifts of their fluorescence spectra towards higher wavelengths at high concentration in urea indicated that the denatured forms of these proteins were less compact and had their tryptophan residues more exposed than their native forms (Creighton, 1980; Schmid, 1989). These results suggested that Bst-TyrRS(wt) and Bst-TyrRS( $\Delta 1)$ were unfolded at high concentration in urea.

The profiles of denaturation with urea for Bst-TyrRS $(\Delta 1)$ and Bst-TyrRS(wt), followed by fluorimetry, had similar parameters and showed two transitions around 3.8 to $4.5 \mathrm{M}$ and 5.6 to $6.2 \mathrm{M}$ (Table 3$)$. These two transitions did not correspond to the successive denaturations of the $C$ and N-terminal domains of Bst-TyrRS(wt), since both existed for Bst-TyrRS $(\Delta 1)$, which included only the N-terminal domain. Could the first transition, around $4 \mathrm{M}$ urea, correspond to the dissociation of native dimeric proteins into folded monomers? The

proteins up to the indicated concentrations. Open symbols represent the values obtained by diluting the proteins, previously denatured in $8 \mathrm{M}$ urea, down to the indicated concentrations of urea. The curves were obtained by fitting the equations described for Table 3 to the data of denaturation (filled symbols). 
Table 3. Parameters of the urea induced transitions with their standard errors in the curve fits (see Figure 7)

\begin{tabular}{lccc}
\hline & Wild-type & $\Delta 1$ & $\Delta 3$ \\
\hline$u_{1}$ & $4.45 \pm 0.23$ & $3.76 \pm 0.06$ & na \\
$c_{1}$ & $1.32 \pm 0.12$ & $1.48 \pm 0.06$ & na \\
$m$ & $0.35 \pm 0.05$ & $0.42 \pm 0.02$ & 0 \\
$u_{2}$ & $6.20 \pm 0.02$ & $5.60 \pm 0.01$ & $6.02 \pm 0.04$ \\
$c_{2}$ & $3.06 \pm 0.22$ & $5.14 \pm 0.19$ & $1.47 \pm 0.08$ \\
\hline
\end{tabular}

The following equation was fit to the experimental data of denaturation, using the program Kaleidagraph 2.1 (Abelbeck Software): $F_{\text {app }}=m /\left(1+\exp \left(c_{1}\left(u_{1}-\right.\right.\right.$ [urea] $\left.\left.)\right)\right)+(1-m) /(1+$ $\exp \left(c_{2}\left(u_{2}-\right.\right.$ [urea] $\left.\left.)\right)\right)$, where $u_{1}$ and $u_{2}$ are the urea concentrations (M) of half-advancement for the first and second transitions of unfolding, $c_{1}$ and $c_{2}$ are their indices of cooperativity and $m$ is the maximal contribution of the first transition to the variation of $F_{\text {app. }}$. We took $m=0$ in the case of Bst-TyrRS( $\left.\Delta 3\right)$ for which we observed only one transition. na, not applicable. The fit of the above equation to the data of Bst-TyrRS(wt) was clearly better for a model with two transitions $\left(\chi^{2}=0.0027\right.$, Pearson's coefficient $R=0.9998)$ than for a model with one transition $\left(\chi^{2}=0.0700\right.$, $R=0.9939)$.

amplitude of this first transition was equal to 35 to $42 \%$ of the total amplitude of the fluorescence signal so that, under this assumption, the same percentage of subunits should be in a free folded form at concentrations of urea between 4.5 and $5.6 \mathrm{M}$. Folded monomers should be more compact and migrate faster than native dimers in gel electrophoresis. However, the profiles of electrophoresis showed only one folded state of Bst-TyrRS(wt) and Bst-TyrRS $(\Delta 1)$ below $5.6 \mathrm{M}$ urea. Thus the first transition corresponded more likely to a conformational change of the dimer than to a dissociation. The cooperativity index of the first transition was low compared with that of the second transition, and suggested that the first transition was due to a conformational change of a region of loose structure, for example the long loop between the $\alpha / \beta$ domain and the $\alpha$-domain, which includes one

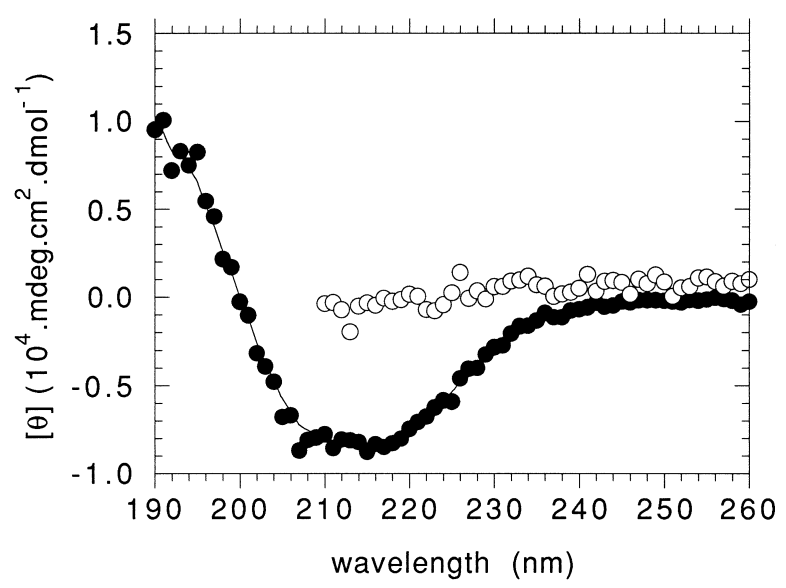

Figure 8. Far-UV circular-dichroism spectra of (0) native and $(\bigcirc)$ unfolded Bst-TyrRS $(\Delta 3)$. The mean residue molar ellipticity [ $\theta$ ] at each wavelength was obtained using the expression $[\theta]=100 \theta_{\mathrm{obs}} / l c$, where $\theta_{\mathrm{obs}}$ is the observed ellipticity in degrees, $c$ is the molar residue concentration and $l$ is the light-path in $\mathrm{cm}$.[ $[\theta]$ varied between -8800 and $+10,000$ deg. $\mathrm{cm}^{2} \cdot \mathrm{dmol}^{-1}$. tryptophan residue. This hypothesis will be tested by mutagenesis.

\section{Swapping of C-terminal domains}

Eco-TyrRS(wt), Bst/Eco-TyrRS, Bst-TyrRS(wt) and Eco/Bst-TyrRS9 tyrosylated E. coli tRNA ${ }^{\mathrm{Tyr}}$ with similar rates at $25^{\circ} \mathrm{C}$ (respectively $100 \%, 41 \%, 36 \%$ and $15 \%$ the rate of Eco-TyrRS(wt), Table 2). These results showed that one could swap the C-terminal domains of Eco-TyrRS and Bst-TyrRS and obtain active hybrid synthetases. They also showed that the C-terminal domains of Eco-TyrRS and Bst-TyrRS were in functional conformations in Eco/Bst-TyrRS9 and Bst/Eco-TyrRS. Thus, either the $\mathrm{N}$ and C-terminal domains of TyrRS do not interact with each other and are independent, or the interactions between them have been at least partially conserved during evolution.

\section{Folding state of a functional C-terminal domain}

The profile of irreversible thermal precipitation for Bst/Eco-TyrRS showed two transitions (Figure 3 and Table 2). The major transition $\left(t_{1 / 2}=51.6^{\circ} \mathrm{C}\right)$ involved $83 \%$ of the molecules and the minor transition $\left(t_{1 / 2}=65.5^{\circ} \mathrm{C}\right)$ involved $17 \%$ of them. In contrast, each of Bst-TyrRS(wt) and Bst-TyrRS $(\Delta 1)$ showed a single transition, around $67.2^{\circ} \mathrm{C}$ and $68.0^{\circ} \mathrm{C}$, respectively. As Bst/Eco-TyrRS, Bst-TyrR$S(w t)$ and Bst-TyrRS $(\Delta 1)$ differed from one another only in their C-terminal domains, the precipitation of $83 \%$ of the Bst/Eco-TyrRS molecules around $51.6^{\circ} \mathrm{C}$ was necessarily due to their C-terminal domain, coming from E. coli. Thus, this C-terminal domain could undergo a transition from an active soluble conformation to a conformation promoting aggregation.

Eco-TyrRS(wt) also showed a single transition, around $48.6^{\circ} \mathrm{C}$. As Bst/Eco-TyrRS and Eco-TyrR$\mathrm{S}(\mathrm{wt})$ differed from one another only in their $\mathrm{N}$-terminal domains, the maintaining of $17 \%$ of the Bst/Eco-TyrRS molecules in a soluble state up to about $65.5^{\circ} \mathrm{C}$ and their precipitation around this temperature were necessarily due to their $\mathrm{N}$-terminal domain coming from B. stearothermophilus. Several explanations can be proposed for the incomplete precipitation of Bst/Eco-TyrRS around $51.6^{\circ} \mathrm{C}$. Part of the molecules could have an unfolded C-terminal domain but remain soluble. They would form $\left[{ }^{14} \mathrm{C}\right]$ tyrosyl adenylate in our titration of the soluble active sites because this reaction depends only on the $\mathrm{N}$-terminal domain. We favour this explanation. Alternatively, part of the molecules could be proteolysed and correspond in fact to the thermostable N-terminal domain of Bst-TyrRS. However, our analysis of the Bst/Eco-TyrRS preparations by gel electrophoresis did not reveal degradation products and therefore do not support this hypothesis (Figure 2).

Four arguments showed that the profiles of thermal precipitation and the corresponding $t_{1 / 2}$ 
values depended only on the structural properties of the tested TyrRSs, even if they were determined on non-purified preparations. The profile of precipitation for the bulk of the E. coli proteins was very different from those for TyrRS and its derivatives (Figures 3 and 5). Bst-TyrRS(wt) precipitated from non-purified preparations with identical profiles when it was expressed from tyrS genes that were carried either by a phagemid or by an M13 phage (Table 2). We have previously shown that Bst-TyrRS(wt) precipitates from purified and non-purified preparations with similar profiles and very close $t_{1 / 2}$ values. We have also studied a family of nine hybrids between the $E$. coli and $B$. stearothermophilus TyrRSs and shown that they precipitate with $t_{1 / 2}$ values that depend on the position of the fusion point (Guez-Ivanier et al., 1993).

The major transition for the thermal precipitation of Bst/Eco-TyrRS had a cooperativity index equal to $0.54 \pm 0.01$. The cooperativity index for the thermal precipitation of Bst-TyrRS $(\Delta 3)$ had a lower index, $0.27 \pm 0.03$. It could be interesting to compare more directly the cooperativities of unfolding for the C-terminal domains of Eco-TyrRS and Bst-TyrRS.

\section{Comparison with previous data and conclusions}

Published data are compatible with a folded state of the C-terminal domain. Bst-TyrRS(wt) and Eco-TyrRS(wt) behave as globular proteins in gel filtration experiments (Dessen et al., 1982; Waye et al., 1983). A proton nuclear magnetic resonance spectrum of Bst-TyrRS(wt) shows only one sharp feature, a pair of doublets (or a triplet) at $1.13 \mathrm{ppm}$, which must arise from one or two aliphatic side-chains (Brick et al., 1989); thus, the C-terminal domain does not move independently of the whole molecule in solution. Incubation of Bst-TyrRS(wt) with trypsin results in the cleavage of the subunit to a protease-resistant fragment of $28 \mathrm{kDa}$, which corresponds to residues 157 to 419, and to smaller fragments (Winter et al., 1979); thus, the C-terminal domain is resistant to proteolysis by trypsin despite the presence of potential cleavage sites in this domain (Winter et al., 1983).

Our results demonstrated that $\operatorname{Bst-TyrRS}(\Delta 3)$, i.e. the isolated C-terminal domain of Bst-TyrRS, was at least partially folded in solution. They indicated that the C-terminal domain of Eco-TyrRS had some structure when it was in an active conformation, since it could undergo a transition to an insoluble conformation. It is therefore likely that the C-terminal domain of Bst-TyrRS has also some structure when it is linked to the $\mathrm{N}$-terminal domain, in its native form. The observation that Bst-TyrRS $(\Delta 3)$ was heat-precipitated around $68^{\circ} \mathrm{C}$ suggests that the C-terminal domain of Bst-TyrRS has some structure at the physiological temperature of growth for B. stearothermophilus. However, several questions remain unanswered. Is the structural state of the isolated C-terminal domain functional and identical with the one that it has in the native Bst-TyrRS? Is the disorder of the C-terminal domain in the crystal structure of Bst-TyrRS due to the flexibility of its peptide link with the $\mathrm{N}$-terminal domain or to a global dynamic variation? Has the isolated C-terminal domain of Bst-TyrRS a well-defined tertiary structure or is it in the molten globule state? Several observations, reported above, encourage us to explore the second hypothesis. This work and, in particular, the possibility of purifying the C-terminal domain of Bst-TyrRS open the way to a detailed structural characterization of this domain and to an analysis of the relations between its disorder and function.

\section{Materials and Methods}

\section{Strains and bacterial techniques}

The plasmids and bacteriophages used in this work are listed in Table 1 . The bacterial strains of E. coli K12, BMH71-18 (supE), HB2151 (supE $E^{+}$) and TG2 (recA, supE), and the media used, LB, M63, $2 \times$ TY, have been described (Carter et al., 1985; Sambrook et al., 1989). The derivatives of TG2 that harboured a recombinant plasmid were grown at $30^{\circ} \mathrm{C}$ because some of these plasmids are toxic at $37^{\circ} \mathrm{C}$ (Bedouelle et al., 1990). TG2 was infected by the derivatives of phage M13 and the infected cells were grown at $37^{\circ} \mathrm{C}$ as described (Vidal-Cros \& Bedouelle, 1992). Soluble extracts were prepared from cultures of strain TG2, either infected by a phage or harbouring a plasmid, as described (Guez-Ivanier et al., 1993).

\section{Constructions of phages M13-B/EY and M13-BY $(\Delta 3)$}

Phage M13-B/EY was constructed by recombining the $B s m I$ restriction sites located at codons 320 to 322 of the Bst-tyrS(BsmI) mutant allele and at codons 324 to 326 of Eco-tyrS ${ }^{+}$, through the following steps. Phage M13mp19(4am) carries a unique BsmI site in its gene 3 and a unique Bam $\mathrm{HI}$ site in its polylinker. The Bam HI fragment of pBR322-EY that carries codons 246 to 423 of Eco-tyrS was purified, then inserted into the BamHI site of M13mp19(4am). The recombinant phage, M13(4am)EY(3'), which carried the 3'-terminal fragment of Eco-tyrS in the same orientation as the lac Z fragment, was identified by digestion with BsmI. The DNAs of phages M13-BY(BsmI) and M13(4am)-EY(3') were digested with $B s m I$, the resulting fragments were ligated, then the recombinant molecules were introduced into strain HB2151 by transformation, which counterselected the phages carrying an amber mutation in gene 4. Phage $\operatorname{M13-BY}(\Delta 3)$ was a gift from Greg Winter. It was constructed by mutagenesis of phage M13-BY(M24) with oligonucleotide 5'GTC GCC GCT AAA CAT GTC CTT CAC3', which deletes codons 2 to 322 of the Bst-tyrS gene (Table 1).

\section{Purification of Bst-TyrRS and its domains}

The production of Bst-TyrRS(wt) and its derivatives from phages M13(4am)-BY(M24.89), M13-BY( $\Delta 1)$ and $\operatorname{M13-BY}(\Delta 3)$, and the first steps of their purifications were performed as described (Vidal-Cros \& Bedouelle, 1992). The soluble extracts $(40 \mathrm{ml}$, prepared from $400 \mathrm{ml}$ of culture at $\left.A_{600 \mathrm{~nm}}=1.0\right)$ were heat-treated then loaded onto a column of DE52 ( $2 \mathrm{ml}$, Whatman). This column was run 
in $50 \mathrm{mM}$ Tris- $\mathrm{HCl}$ (pH 7.5), $10 \mathrm{mM}$ 2-mercaptoethanol, $0.1 \mathrm{mM}$ phenylmethylsulfonide fluoride (PMSF) and $\mathrm{NaCl}$. For the purification of Bst-TyrRS(wt), the column was washed with $10 \mathrm{ml}$ of $0 \mathrm{mM} \mathrm{NaCl}, 10 \mathrm{ml}$ of $50 \mathrm{mM}$ $\mathrm{NaCl}$, then eluted with $150 \mathrm{mM} \mathrm{NaCl}$. For Bst-TyrRS $(\Delta 1)$, it was washed with $0 \mathrm{mM} \mathrm{NaCl}, 20 \mathrm{mM} \mathrm{NaCl}$, then eluted with $100 \mathrm{mM} \mathrm{NaCl}$. Bst-TyrRS(wt) and Bst-TyrRS( $\Delta 1)$ were further purified by two successive chromatographies on a Mono $\mathrm{Q}$ column (Pharmacia) at different $\mathrm{pH}$ values, as described (First \& Fersht, 1993). Bst-TyrRS( $\Delta 3$ ) did not bind to the DE52 column in the above conditions ( $\mathrm{pH}$ 7.5), contrary to most proteins of the soluble extract. The fraction flowing through the column was concentrated using a Centricon 3 cartridge (Amicon) and dialysed against $50 \mathrm{mM}$ Tris- $\mathrm{HCl}(\mathrm{pH}$ 8.6), $5 \mathrm{mM}$ 2-mercaptoethanol, $0.1 \mathrm{mM}$ PMSF. Bst-TyrRS( $\Delta 3)$ was further purified by chromatography on a Mono $Q$ column in the same buffer ( $\mathrm{pH} 8.6)$ and eluted at $120 \mathrm{mM} \mathrm{NaCl}$. The purified proteins were more than $90 \%$ pure as judged by gel electrophoresis. They were kept frozen at $-70^{\circ} \mathrm{C}$, in $20 \mathrm{mM}$ Tris- $\mathrm{HCl}$ (pH 7.78), $5 \mathrm{mM}$ 2-mercaptoethanol.

\section{Activity and concentration measurements}

The concentration of TyrRS active sites in the soluble extracts and the initial rate of tyrosylation of crude $E$. coli tRNA by these extracts were measured as described (Guez-Ivanier et al., 1993). The concentrations of purified proteins were measured with the Bio-Rad reagent and bovine serum albumin as the standard. The intensities of protein bands in Western blots were quantified with an image analyser (Masterscan from Scanalytics, USA).

\section{Thermal precipitation}

The cellular extracts were divided into identical portions, which were simultaneously incubated at varying temperatures for 30 minutes. The samples were cooled for ten minutes on ice and the precipitate of protein was eliminated by centrifugation. The concentration of TyrRS active sites in the unheated samples was around $1 \mu \mathrm{M}$. Several control experiments were performed, as previously reported (Guez-Ivanier et al., 1993).

\section{Gel electrophoresis and Western blotting}

The ratio of acrylamide to bisacrylamide was 29:1 $(\mathrm{w} / \mathrm{w})$ in the SDS-containing gels (Laemmli, 1970). The urea gradient gels $(8 \mathrm{~cm} \times 10 \mathrm{~cm} \times 0.15 \mathrm{~cm})$ contained a gradient of urea ( 0 to $8 \mathrm{M}$ ), a compensatory gradient of polyacrylamide (either 10 to $7.5 \%$ or 15 to $11 \%, \mathrm{w} / \mathrm{v}$ ) and a ratio of acrylamide to bisacrylamide of 30:0.8 (Goldenberg, 1989). They were run in a buffer containing $0.2 \mathrm{M}$ glycine, $25 \mathrm{mM}$ Tris- $\mathrm{OH}$ ( $\mathrm{pH} 8.4$ ), in an apparatus cooled by running water, during 220 to 250 minutes at $12 \mathrm{~mA}$ depending on the acrylamide concentration. The $\mathrm{pH}$ of the buffer was higher than the $\mathrm{pI}$ values of Bst-TyrRS(wt), Bst-TyrRS( $\Delta 1)$ and Bst-TyrRS( $\Delta 3): 5.6,5.4$ and 7.7, respectively, estimated from their amino acid sequences. After electrophoresis, the proteins were either stained in the gels with Coomassie blue, or transferred from the gels to nitrocellulose sheets (Hybond C, Amersham) by Western blotting. In this last case, the immobilized proteins were revealed by immunostaining with a rabbit antiserum directed against Bst-TyrRS and a goat antibody, directed against the Fc fragment of rabbit immunoglobulins $\mathrm{G}$ and conjugated with alkaline phosphatase, as described (Sambrook et al., 1989). The anti-TyrRS serum was prepared by injecting rabbits three times with $1 \mathrm{mg}$ of purified Bst-TyrRS in $1 \mathrm{ml}$ of Freund adjuvant, at two to three-week intervals.

\section{Fluorimetric measurement of urea denaturation}

Denaturation with urea was performed as described (Pace et al., 1989). Each reaction mixture contained $50 \mathrm{mM}$ Tris-HCl, $5 \mathrm{mM}$ 2-mercaptoethanol, 0.1 mM PMSF, varying concentrations of urea at $\mathrm{pH} 7.78$ and $10 \mu \mathrm{g} / \mathrm{ml}$ protein. The solutions of urea (Schwarz-Mann, ICN) were prepared daily. The final concentration of urea in each sample was checked by measuring the refractive index. The protein solutions were incubated for 12 hours at $25^{\circ} \mathrm{C}$ to let the reactions of denaturation and renaturation reach equilibrium, except when otherwise indicated. The proteins were denatured during three hours in $8 \mathrm{M}$ urea then diluted to the final concentration of urea, for the experiments of reversibility. The fluorescence measurements were performed at $25^{\circ} \mathrm{C}$ as described (Schmid, 1989), using a Perkin-Elmer LS5B apparatus, with slit-widths of $2.5 \mathrm{~nm}$ for the excitation light and $5 \mathrm{~nm}$ for emission. The signal was averaged during 17 seconds. The signal of the solvent was subtracted from the signal of the protein sample at each concentration of urea.

\section{Circular dichroism spectra}

The far-UV circular dichroism spectra were recorded as described (Guijarro et al., 1995), with the following modifications. The increment of wavelength was $1 \mathrm{~nm}$, the accumulation time was two seconds per step and the temperature was $20^{\circ} \mathrm{C}$. The sample of $\operatorname{Bst-TyrRS}(\Delta 3)$ contained $0.2 \mathrm{mg} / \mathrm{ml}(21 \mu \mathrm{M})$ of protein, $50 \mathrm{mM}$ Tris- $\mathrm{HCl}$ $(\mathrm{pH} 7.78)$ and $5 \mathrm{mM}$ 2-mercaptoethanol. It contained $0 \mathrm{M}$ urea for the native conditions and had been equilibrated for three hours in $7.5 \mathrm{M}$ urea for the denaturing conditions. The signal of the solvent was substracted from the signal of the protein sample in each of the experimental conditions. The content in secondary structures was estimated as described (Hennessey \& Johnson, 1981; Manavalan \& Johnson, 1987), with a data base of 32 spectra.

\section{Acknowledgements}

We thank Greg Winter for the gift of phage $\operatorname{M13-BY}(\Delta 3)$, Anne Vidal-Cros and François Brégégère for raising the serum against tyrosyl-tRNA synthetase, Thierry Rose and Yvonne Guillou for their help in the CD measurements, Thierry Rose for the analysis of the CD data, Michel Goldberg and Roger Pain for useful discussions. V.G.-I. was the recipient of a Roux fellowship. This work was supported in part by grants from the Interface Chimie-Biologie of CNRS.

\section{References}

Avis, J. M., Day, A. G., Garcia, G. A. \& Fersht, A. R. (1993). Reaction of modified and unmodified tRNA $^{\mathrm{Tyr}}$ substrates with tyrosyl-tRNA synthetase (Bacillus stearothermophilus). Biochemistry, 32, 5312-5320.

Barker, D. G. (1982). Cloning and amplified expression of the tyrosyl-tRNA synthetase genes of Bacillus 
stearothermophilus and Escherichia coli. Eur. J. Biochem. 125, 357-360.

Bedouelle, H. \& Winter, G. (1986). A model of synthetase/transfer RNA interaction as deduced by protein engineering. Nature, 320, 371-373.

Bedouelle, H., Guez, V., Vidal-Cros, A. \& Hermann, M. (1990). Overproduction of tyrosyl-tRNA synthetase is toxic to Escherichia coli: a genetic analysis. J. Bacteriol. 172, 3940-3945.

Bedouelle, H., Guez-Ivanier, V. \& Nageotte, R. (1993). Discrimination between transfer-RNAs by tyrosyltRNA synthetase. Biochimie, 75, 1099-1108.

Beese, L. S., Derbyshire, V. \& Steitz, T. A. (1993). Structure of DNA polymerase I Klenow fragment bound to duplex DNA. Science, 260, 352-355.

Bradford, M. M. (1976). A rapid and sensitive method for the quantitation of microgram quantities of protein utilizing the principle of protein-dye binding. Anal. Biochem. 72, 248-254.

Brick, P., Bhat, T. N. \& Blow, D. M. (1989). Structure of tyrosyl-tRNA synthetase refined at $2.3 \AA$ resolution. Interaction of the enzyme with the tyrosyl-adenylate intermediate. J. Mol. Biol. 208, 83-98.

Carter, P., Bedouelle, H. \& Winter, G. (1985). Improved oligonucleotide site-directed mutagenesis using M13 vectors. Nucl. Acids Res. 13, 4431-4443.

Carter, P., Bedouelle, H. \& Winter, G. (1986). Construction of heterodimer tyrosyl-tRNA synthetase shows tRNA $^{\mathrm{Tyr}}$ interacts with both subunits. Proc. Natl Acad. Sci. USA, 83, 1189-1192.

Creighton, T. E. (1980). Kinetic study of protein unfolding and refolding using urea gradient electrophoresis. J. Mol. Biol. 137, 61-80.

Dente L. \& Cortese R. (1987). pEMBL: a new family of single-stranded plasmids for sequencing DNA. Methods Enzymol. 155, 111-119.

Dessen, P., Zaccaï, G. \& Blanquet, S. (1982). Neutron scattering studies of Escherichia coli tyrosyl-tRNA synthetase and of its interaction with tRNA ${ }^{\mathrm{Tyr}}$. J. Mol. Biol. 159, 651-664.

Fersht, A. R. (1987). Dissection of the structure and activity of the tyrosyl-tRNA synthetase by sitedirected mutagenesis. Biochemistry, 26, 8031-8037.

First, E. A. \& Fersht, A. R. (1993). Mutation of lysine 233 to alanine introduces positive cooperativity into tyrosyl-tRNA synthetase. Biochemistry, 32, 1365113657.

Frankel, A. D. (1992). The importance of being flexible. Proc. Natl Acad. Sci. USA, 89, 11653.

Frankel, A. D. \& Kim, P. S. (1991). Modular structure of transcription factors: implications for gene regulation. Cell, 65, 717-719.

Goldenberg, D. P. (1989). Analysis of protein conformation by gel electrophoresis. In Protein Structure, a Practical Approach (Creighton, T. E., ed), pp. 225-250, IRL Press, Oxford.

Guez-Ivanier, V., Hermann, M., Baldwin, D. \& Bedouelle, H. (1993). Mapping the stability determinants of bacterial tyrosyl transfer RNA synthetases by an experimental evolutionary approach. J. Mol. Biol. 234, 209-221.

Guijarro, J. I., Jackson, M., Chaffotte, A. F., Delepierre, M., Mantsch, H. H. \& Goldberg, M. E. (1995). Protein folding intermediates with rapidly exchangeable amide protons contain authentic hydrogen-bonded secondary structures. Biochemistry, 34, 2998-3008.

Hennessey, J. P., Jr \& Johnson, W. C., Jr (1981). Information content in the circular dichroism of proteins. Biochemistry, 20, 1085-1094.

Hollecker, M. \& Creighton, T. E. (1982). Effect on protein stability of reversing the charge on amino groups. Biochim. Biophys. Acta, 701, 395-404.

Jardetzky, O., Akasaka, K., Vogel, D., Morris, S. \& Holmes, K. C. (1978). Unusual segmental flexibility in a region of tobacco mosaic virus coat protein. Nature, 273, 564-566.

Laemmli, U. K. (1970). Cleavage of structural proteins during the assembly of the head of bacteriophage T4. Nature, 227, 680-685.

Manavalan, P. \& Johnson, W. C., Jr (1987). Variable selection method improves the prediction of protein secondary structure from circular dichroism spectra. Anal. Biochem., 167, 76-85.

Munowitz, M. G., Dobson, C. M., Griffin, R. G. \& Harrison, S. C. (1980). On the rigidity of RNA in tomato bushy stunt virus. J. Mol. Biol. 141, 327333.

Pace, C. N., Shirley, B. A. \& Thomson, J. A. (1989). Measuring the conformational stability of a protein. In Protein Structure, a Practical Approach (Creighton, T. E., ed.), pp. 311-330, IRL Press, Oxford.

Sambrook, J., Fritsch, E. F. \& Maniatis, T. (1989). Molecular Cloning: A Laboratory Manual. Cold Spring Harbor Laboratory Press, Cold Spring Harbor, N.Y.

Schmid, F. X. (1989). Spectral methods of characterizing protein conformation and conformational changes. In Protein Structure, a Practical Approach (Creighton, T. E., ed.), pp. 251-285, IRL Press, Oxford.

Schimmel, P., Giegé, R., Moras, D. \& Yokoyama, S. (1993). An operational RNA code for amino acids and possible relationship to genetic code. Proc. Natl Acad. Sci. USA, 90, 8763-8768.

Vidal-Cros, A. \& Bedouelle, H. (1992). Role of residue Glu152 in the discrimination between transfer RNAs by tyrosyl-tRNA synthetase from Bacillus stearothermophilus. J. Mol. Biol. 223, 801-810.

Waye, M. M. Y. \& Winter, G. (1986) A transcription terminator in the $5^{\prime}$ non-coding region of the tyrosyl-tRNA synthetase gene from Bacillus stearothermophilus. Eur. J. Biochem. 158, 505-510.

Waye, M. M. Y., Winter, G., Wilkinson, A. J. \& Fersht, A. R. (1983). Deletion mutagenesis using an 'M13 splint': the N-terminal structural domain of tyrosyltRNA synthetase (B. stearothermophilus) catalyses the formation of tyrosyl adenylate. EMBO J. 2, 18271829.

Winter, G., Koch, G. L. E., Dell, A. \& Hartley, B. S. (1979). The tryptophanyl- and tyrosyl-tRNA synthetases from Bacillus stearothermophilus. In Transfer RNA: Structure, Properties, and Recognition, pp. 255-265, Cold Spring Harbor Laboratory Press, Cold Spring Harbor, NY.

Winter, G., Koch, G. L. E., Hartley, B. S. \& Barker, D. G. (1983). The amino acid sequence of the tyrosyl-tRNA synthetase from Bacillus stearothermophilus. Eur. J. Biochem. 132, 383-387.

Edited by A. R. Fersht 